\title{
Design of Metronome Based on AT89C51 Microcontroller
}

\author{
Ting Zhang
}

College of Information Engineering, Xijing University, Shanxi Xian 710123

\section{Keywords: SCM; Metronome; Digital tube; Simulation}

\begin{abstract}
The metronome is a device that produces beats at regular intervals and can be used as the reference of music's speed. It has a wide application in music. The metronome was designed based on the AT89C51 microcontroller. The peripheral circuit is mainly composed of digital display module, keyboard module and voice output module. The utility model has the functions of stable rhythm, adjustable speed and real-time display. We carried out the simulation in the PROTUES and the results show that the metronome has a high accuracy and is easy to control parameters.
\end{abstract}

\section{Introduction}

With the development of society, the demand of people for entertainment agencies and entertainment equipment has become increasingly higher and higher, changing from mechanical equipment to automation and intelligence, which can better control and more convenient in entertainment. As an indispensable entertainment tool, electronic music metronome will become a necessary entertainment equipment to replace the artificial beat which can be more accurate and more convenient according to different music to match the rhythm. Seen from a different point of view, electronic music is more reliable and appreciative than the beat of the artificial beat. Therefore, a metronome based on AT89C51 is designed.

\section{Working Principle}

AT89C51 is the core control device of metronome which is composed of digital display module, keyboard control module and audio output module, the diagram is shown in fig. 1.

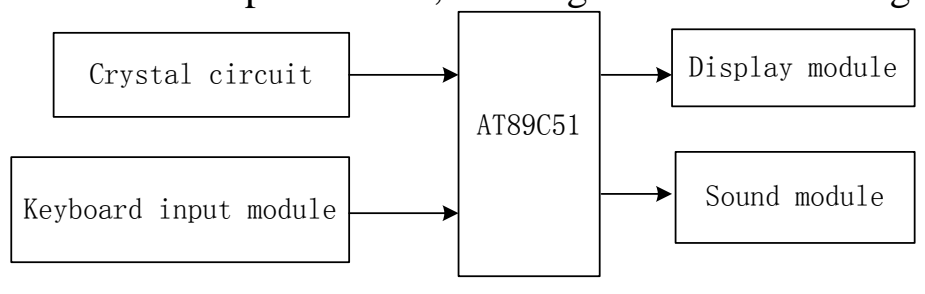

Figure 1. The principle diagram of metronome

The display module is composed of four in one LED display, and the dynamic scanning display mode is adopted. The keyboard adjustment module includes the function selection key, the parameter selection key, the parameter increment key and the parameter reduction key. The sound output module is composed of a power amplifying circuit, and the output pulse of different frequency generated by a single chip microcomputer is amplified by power amplification circuit to drive the speaker.

The Hardware Design of Metronome. The metronome is mainly composed of hardware and software two parts, and AT89C51 is the control module. The peripheral circuit comprises a clock circuit, reset circuit, MCU, digital display module and keyboard button circuit which is shown in fig. 2 . 


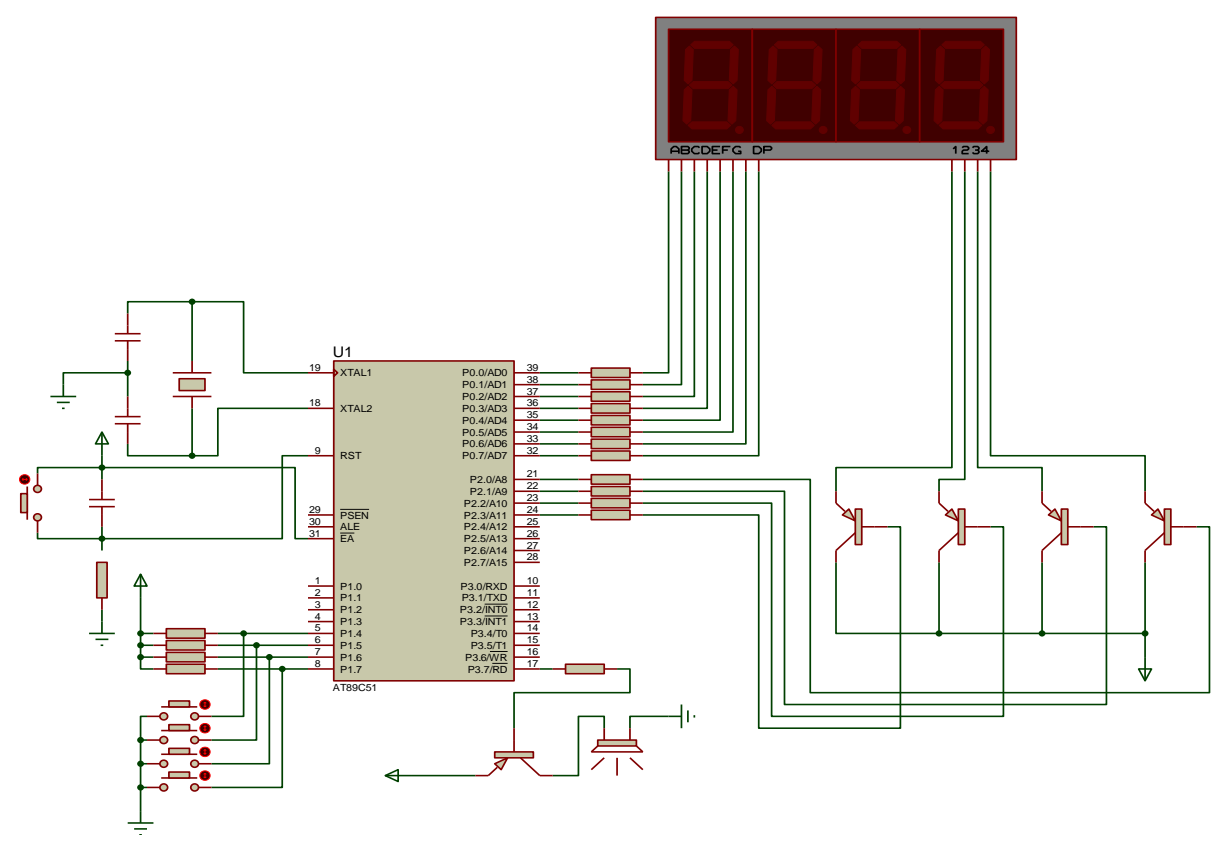

Figure 2. The circuit principle diagram metronome

P3.7 port outputs the beat pulse to control Q6 (8550 PNP) to conduct and the buzzer will sound. The digital display module consists of four Yang digital tube and four (8550 PNP) transistors which were selected respectively by P2.0 P2.3 four ports to achieve dynamic scanning, P0 outputs display driving signal. The keyboard module comprises switch S1, S2, S3, S4, S5, where, S1 is select key of metronome and rhythm parameters, S2 is the parameter select key, S3 is the increasing key, S4 is reduction key,S5 is the reset button. After MCU responses interrupt, speed of the beat is increased or decreased by the program.

The Software Design of Metronome. The system software adopts the modular structure design which includes the main program module, keyboard identification module, digital tube display module and sound module and the main program flow chart is shown in fig. 3.

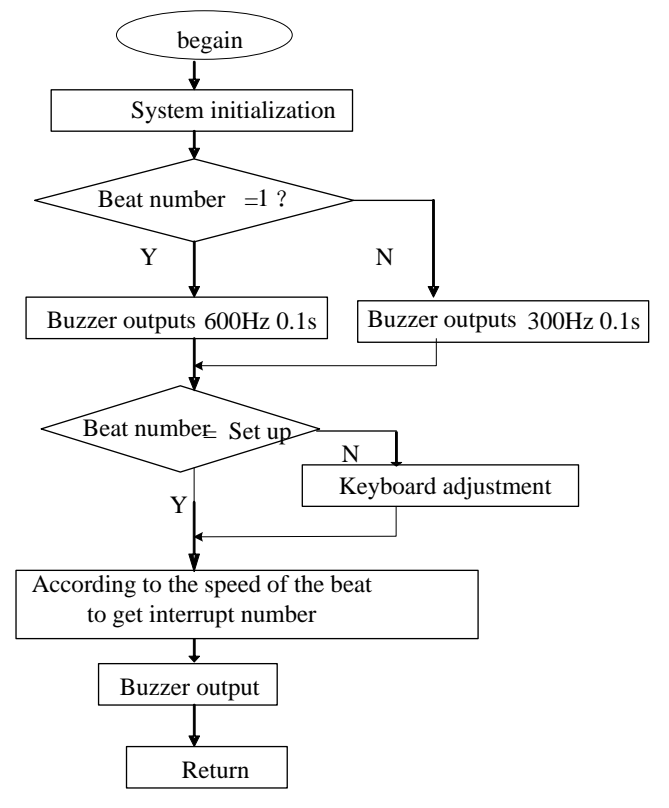

Figure 3 The flow chart of main program 


\section{The Simulation and Result Analysis}

System simulation parameters: the speeds range is $24 \sim 400 / \mathrm{min}$ which is adjustable, beat error is less than 1\%; beat number is $1 \sim 9$ which is adjustable. The liquid crystal displays beat number and the loudspeaker beats. It generates $600 \mathrm{~Hz}$ high frequency sounds lasting $0.1 \mathrm{~s}$ when the beat number is one. It generates $300 \mathrm{~Hz}$ low frequency sound lasting $0.1 \mathrm{~s}$ when displays other beat number. The experimental results are obtained by the simulation in the PROTUES software shown in fig. 4.

When supply the system with the power, the highest position of the four digital tubes displays the number of beats, the latter three display speed of the beat, the overall display 1024, and the buzzer is in the cut-off state shown in fig. 4.

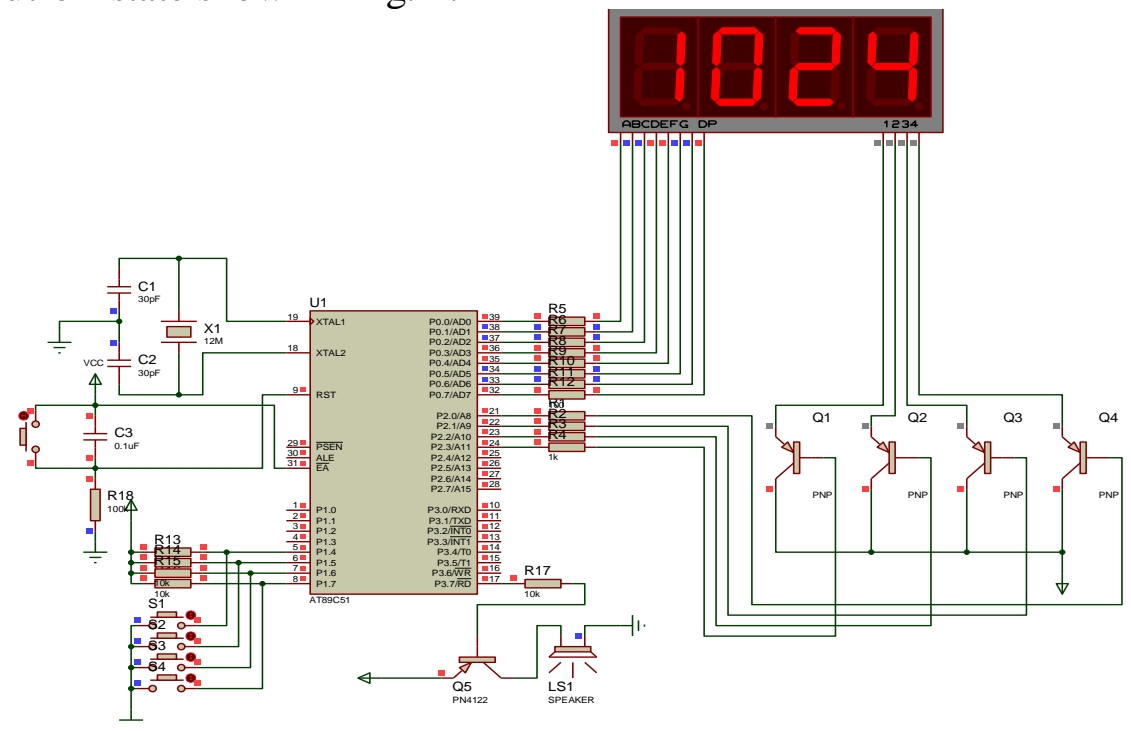

Figure 4. The initial state of metronome

The first time you press the switch S1, metronome is parameter adjustment function, The first time you press the switch S2, the speed is adjusted, the second time you press switch S2, the number of beats can adjusted. At the time, speed of beat 66 is beat $/ \mathrm{min}$, beat number 3 (voice frequency is $300 \mathrm{~Hz}$ ), adjusted result is shown in Fig. 5

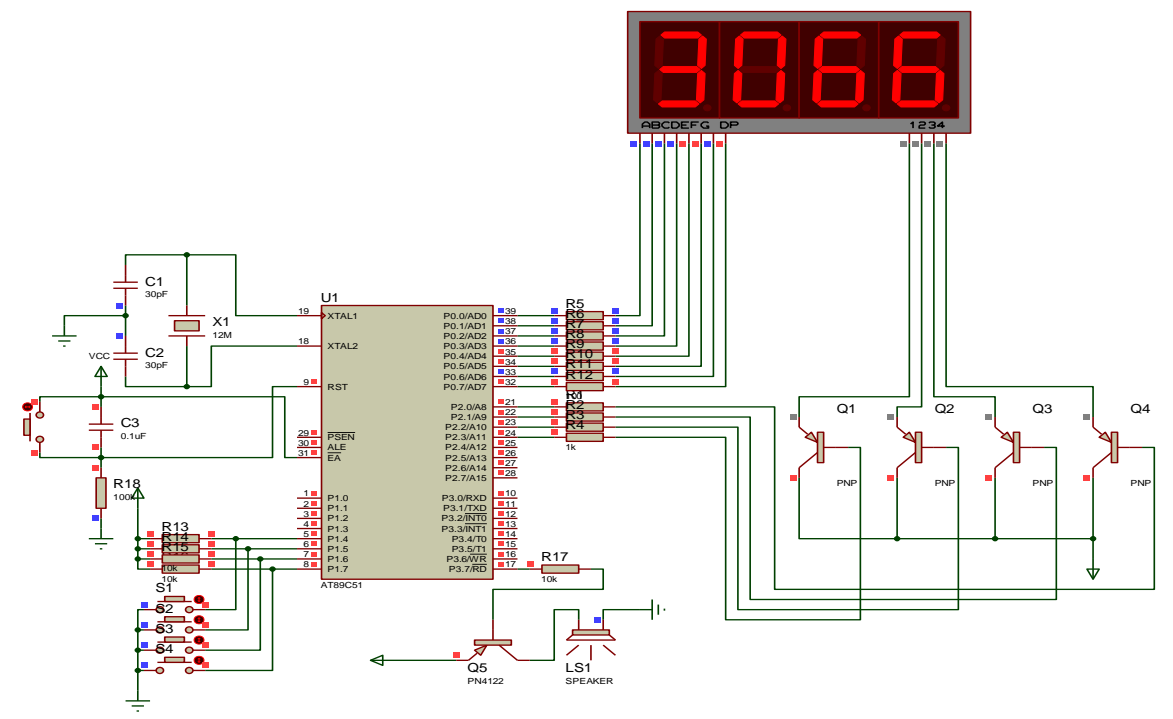

Figure 5. parameter adjustment state

After adjust the beat number and beat speed, when you press the switch S1second time, the metronome is beat function, buzzer beats, four digital tube display beat number. When it is 3 and the metronome outputs beats with $300 \mathrm{~Hz}$ shown in fig. 6 . 


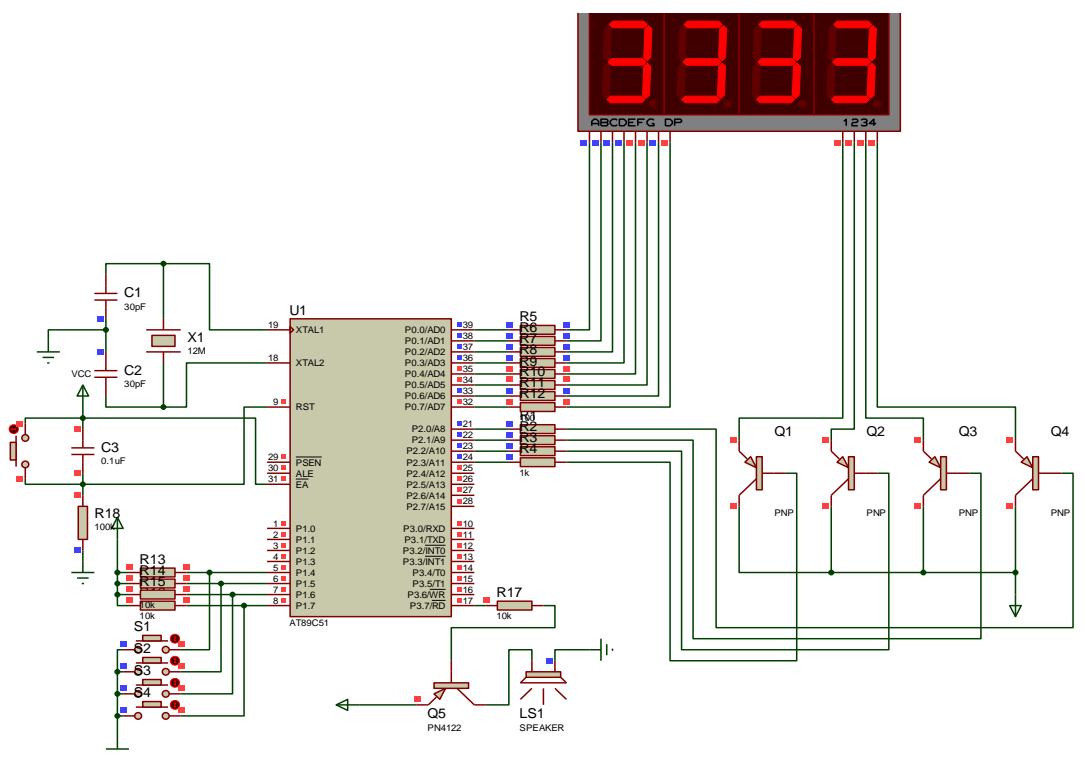

Figure 6. Beat output status

\section{Conclusions}

A electronic metronome is designed based on interrupt and timing system of AT89C51, the simulation results verify the metronome has such advantages as table rhythm, speed adjustable, graceful timbre features and users can achieve speed accurately preset through the keyboard input. The control circuit is simple and easy to realize.

\section{Acknowledgements}

It is supported by the Research Fund Project of Xijing University (XJ160228).

\section{References}

[1] Yan Chengjian, Tong Zhongliang. Basic Music Theory Tutorial [M].Beijing: People's Music Publishing House, 2006.

[2] Zhao Yune, Huang Jie. MIDI Generator design based on H6188 Voice IC [J]. Electronic Technology,2016,(02):60-61+50.

[3] Qin Yumeng, Wang Yanan, Qiu Chunling. Design and Implementation of An Efficient Software Timer [J]. Research and Exploration in Laboratory,2015,(09):94-97+106.

[4] Bai Yanxiang. Design and Development of Remote Control System in Embedded Marine Main Engine [D].Dalian Maritime University,2015.

[5] Yang Jianqing. System Design and Implementation of Automatic Frequency Correction Based on Direct Frequency Measurement [D].National Time Service Center (Chinese Academy of Sciences), 2015.

[6] Du Changqing, Pan Zhiqiang, Zhao Yifan, Zhu Yiduo, Yao Hua. Research on Charging System of Electric Vehicle [J].Chinese Journal of Power Sources,2016,(06):1277-1279+1329.

[7] Long Jun. Metronome Application in Music Teaching in the College Entrance Examination [J].Music Space, 2015,(11):173+184.

[8] Huang Sufang, Chen Yuanyuan, Yan Li, Lu Ji Di, Zou Dengxiu. Feasibility Study on Frequency of Chest Compressions Guided by Musical Metronome [J].Chinese Nursing Research,2014,(02):194-195. 
[9] Ma Jianhui, Wang Zhiyou, Zhuang Ruke, Liu Yuan. Design and Implementation of An Efficient Timer Module [J].Qutlook of Electronic Technology,2011,(03):31-32+39.

[10] Dai Luwei. Timer Design with Long Time and High-Precision Based on Embedded ARM [D].Donghua University,2015. 